\title{
Existence and Iteration of Positive Solutions to Third-Order BVP for a Class of $p$-Laplacian Dynamic Equations on Time Scales
}

\author{
A. Kameswara Rao \\ Department of Mathematics, Gayatri Vidya Parishad College of Engineering for Women, Madhurawada, \\ Visakhapatnam 530 048, India
}

Correspondence should be addressed to A. Kameswara Rao; kamesh_1724@yahoo.com

Received 8 August 2015; Revised 27 October 2015; Accepted 16 November 2015

Academic Editor: Patricia J. Y. Wong

Copyright (C) 2015 A. Kameswara Rao. This is an open access article distributed under the Creative Commons Attribution License, which permits unrestricted use, distribution, and reproduction in any medium, provided the original work is properly cited.

We investigate the existence and iteration of positive solutions for the following third-order $p$-Laplacian dynamic equations on time scales: $\left(\phi_{p}\left(u^{\Delta \Delta}(t)\right)\right)^{\nabla}+q(t) f\left(t, u(t), u^{\Delta \Delta}(t)\right)=0, t \in[a, b], \alpha u(\rho(a))-\beta u^{\Delta}(\rho(a))=0, \gamma u(b)+\delta u^{\Delta}(b)=0, u^{\Delta \Delta}(\rho(a))=0$, where $\phi_{p}(s)$ is $p$-Laplacian operator; that is, $\phi_{p}(s)=|s|^{p-2} s, p>1, \phi_{p}^{-1}=\phi_{q}$, and $1 / p+1 / q=1$. By applying the monotone iterative technique and without the assumption of the existence of lower and upper solutions, we not only obtain the existence of positive solutions for the problem, but also establish iterative schemes for approximating the solutions.

\section{Introduction}

A time scale $\mathbb{T}$ is a nonempty closed subset of $\mathbb{R}$. We make the blanket assumption that $a, b$ are points in $\mathbb{T}$. By an interval $[a, b]$, we always mean the intersection of the real interval $[a, b]$ with the given time scale; that is, $[a, b] \cap \mathbb{T}$.

In recent years, dynamic equations on time scales have found a considerable interest and attracted by many researchers; see, for example, [1-11]. The reason seems to be twofold. Theoretically, dynamic equations on time scales not only can unify differential and difference equations [12], but also have displayed much more complicated dynamics $[13,14]$. Moreover, the study of time scales has led to several important applications in the study of insect population models, neural networks, stock market, heat transfer, economic, wound healing, and epidemic models.

Recently, there is much attention paid to the existence of positive solutions for second-order nonlinear boundary value problems on time scales; see $[2,6,15,16]$ and the references therein. On the one hand, higher-order nonlinear boundary value problems have been studied extensively; for details, see $[4,10,17,18]$ and references therein. On the other hand, the boundary value problems with $p$-Laplacian operator have also been discussed extensively in the literature; for example, see $[3,7-9,11,19-21]$. However, to the best of our knowledge, there are not many results concerning the third-order $p$ Laplacian dynamic equations on time scales.

In [22], Yang and Yan studied the following third-order Sturm-Liouville boundary value with $p$-Laplacian:

$$
\begin{aligned}
\left(\phi_{p}\left(u^{\prime \prime}(t)\right)\right)^{\prime}+f(t, u(t)) & =0, \quad t \in(0,1), \\
\alpha u(0)-\beta u^{\prime}(0) & =0, \\
\gamma u(0)+\delta u^{\prime}(1) & =0, \\
u^{\prime \prime}(0) & =0 .
\end{aligned}
$$

By using the fixed point index method, they established the existence of at least one or two positive solutions for the third-order Sturm-Liouville boundary value problem with $p$ Laplacian. 
In [8], Han and Kang were concerned with the existence of multiple positive solutions of third-order $p$-Laplacian dynamic equation on time scales:

$$
\begin{aligned}
\left(\phi_{p}\left(u^{\Delta \Delta}(t)\right)\right)^{\nabla}+f(t, u(t)) & =0, \quad t \in[a, b], \\
\alpha u(\rho(a))-\beta u^{\Delta}(\rho(a)) & =0 \\
\gamma u(b)+\delta u^{\Delta}(b) & =0 \\
u^{\Delta \Delta}(\rho(a)) & =0
\end{aligned}
$$

where $\phi_{p}(s)=|s|^{p-2} s, p>1$. By using the fixed point index method, they obtained the existence of multiple positive solutions for singular nonlinear boundary value problem.

In this paper, we are concerned with the existence and iteration of positive solutions to the following third-order $p$ Laplacian dynamic equation on time scales:

$$
\begin{aligned}
\left(\phi_{p}\left(u^{\Delta \Delta}(t)\right)\right)^{\nabla}+q(t) f\left(t, u(t), u^{\Delta \Delta}(t)\right) & =0, \\
t & \in[a, b], \\
\alpha u(\rho(a))-\beta u^{\Delta}(\rho(a)) & =0, \\
\gamma u(b)+\delta u^{\Delta}(b) & =0, \\
u^{\Delta \Delta}(\rho(a)) & =0,
\end{aligned}
$$

where $\phi_{p}(s)$ is $p$-Laplacian operator; that is, $\phi_{p}(s)=|s|^{p-2} s$ for $p>1$ with $\phi_{p}^{-1}=\phi_{q}$ and $1 / p+1 / q=1 ; \alpha, \beta, \gamma, \delta \geq 0$.

Throughout this paper, we assume that the following conditions are satisfied:

(H1) $d=\gamma \beta+\alpha \delta+\alpha \gamma(b-\rho(a))>0$.

(H2) $q(t)$ is a nonnegative continuous function defined on $(\rho(a), b)$, and $q(t) \neq \equiv$ on any subinterval of $(\rho(a), b)$. In addition, $\int_{\rho(a)}^{b} q(t) \nabla t<+\infty$. Here, a positive solution of (3) and (4) means a solution $u^{*}$ satisfying $u^{*}(t)>0, \rho(a)<t<b$.

(H3) $f:[\rho(a), b] \times[0, \infty) \times \mathbb{R} \rightarrow[0, \infty)$ is continuous, and there exist constants $\mu_{1}, \mu_{2}>0$, such that, for any $t \in[\rho(a), b], u \in[0,+\infty), v \in \mathbb{R}$,

$$
\begin{array}{ll}
f(t, \lambda u, v) \geq \lambda^{\mu_{1}} f(t, u, v), & \forall 0 \leq \lambda \leq 1, \\
f(t, u, \lambda v) \geq \lambda^{\mu_{2}} f(t, u, v), & \forall 0 \leq \lambda \leq 1 .
\end{array}
$$

(H4) $f(t, 0,0) \not \equiv 0$ and $f(t, 1,1) \not \equiv 0$, and let

$$
k=\max _{t \in[\rho(a), b]} f(t, 1,1) .
$$

Remark 1. Inequalities (5) and (6) are equivalent to the following inequalities, respectively:

$$
\begin{array}{ll}
f(t, \lambda u, v) \leq \lambda^{\mu_{1}} f(t, u, v), & \forall \lambda \geq 1, \\
f(t, u, \lambda v) \leq \lambda^{\mu_{2}} f(t, u, v), & \forall \lambda \geq 1 .
\end{array}
$$

This paper is organized as follows. In Section 2, we state some basic time scale definitions and several preliminary results. Section 3 is devoted to the iteration and existence of positive solutions of for the third-order Sturm-Liouville boundary value problem with $p$-Laplacian on time scales (3)(4). Finally, in Section 4, we give an example to demonstrate our main result.

\section{Preliminaries}

For convenience, we list the following definitions which can be found in $[3,5,13-15,23]$.

Definition 2. A time scale $\mathbb{T}$ is a nonempty closed subset of real numbers $\mathbb{R}$. For $t<\sup \mathbb{\mathbb { V }}$ and $t>\inf \mathbb{\mathbb { T }}$, define the forward jump operator $\sigma$ and backward jump operator $\rho$, respectively, by

$$
\begin{aligned}
& \sigma(t)=\inf \{\tau \in \mathbb{T} \mid \tau>t\} \in \mathbb{T}, \\
& \rho(r)=\sup \{\tau \in \mathbb{T} \mid \tau<r\} \in \mathbb{T},
\end{aligned}
$$

for all $t, r \in \mathbb{T}$. If $\sigma(t)>t, t$ is said to be right scattered, and if $\rho(r)<r, r$ is said to be left scattered; if $\sigma(t)=t, t$ is said to be right dense, and if $\rho(r)=r, r$ is said to be left dense. If $\mathbb{T}$ has a right scattered minimum $m$, define $\mathbb{T}_{k}=\mathbb{T}-\{m\}$; otherwise set $\mathbb{T}_{k}=\mathbb{T}$. If $\mathbb{T}$ has a left scattered maximum $M$, define $\mathbb{T}^{k}=\mathbb{T}-\{M\}$; otherwise $\mathbb{T}^{k}=\mathbb{T}$.

Throughout this paper, we make the blanket assumption that $a \leq b$ are points in $\mathbb{T}$ and define the interval in $\mathbb{T}$ :

$$
[a, b]=\{t \in \mathbb{T} \text { such that } a \leq t \leq b\} .
$$

Other types of intervals are defined similarly.

Definition 3. For $f: \mathbb{T} \rightarrow \mathbb{R}$ and $t \in \mathbb{T}^{k}$, the delta derivative of $f$ at the point $t$ is defined to be the number $f^{\Delta}(t)$ (provided it exists) with the property that, for each $\epsilon>0$, there is a neighborhood $U$ of $t$ such that

$$
\left|f(\sigma(t))-f(s)-f^{\Delta}(t)(\sigma(t)-s)\right| \leq \epsilon|\sigma(t)-s|,
$$

for all $s \in U$.

For $f: \mathbb{T} \rightarrow \mathbb{R}$ and $t \in \mathbb{T}_{k}$, the nabla derivative of $f$ at $t$ is denoted by $f^{\nabla}(t)$ (provided it exists) with the property that, for each $\epsilon>0$, there is a neighborhood $U$ of $t$ such that

$$
\left|f(\rho(t))-f(s)-f^{\nabla}(t)(\rho(t)-s)\right| \leq \epsilon|\rho(t)-s|,
$$

for all $s \in U$.

Definition 4. A function $f$ is left-dense-continuous (i.e., ldcontinuous), if $f$ is continuous at each left-dense point in $\mathbb{T}$ and its right-sided limit exists at each right-dense point in $\mathbb{T}$. It is well known that if $f$ is ld-continuous, then there is a function $F(t)$ such that $F^{\nabla}(t)=f(t)$. In this case, it is defined that

$$
\int_{a}^{b} f(t) \nabla t=F(b)-F(a) .
$$


Definition 5. If $G^{\Delta}(t)=f(t)$, then we define the delta integral by

$$
\int_{a}^{b} f(t) \Delta t=G(b)-G(a) .
$$

If $F^{\nabla}(t)=f(t)$, then we define the nabla integral by

$$
\int_{a}^{b} f(t) \nabla t=F(b)-F(a) .
$$

If $u^{\Delta \Delta}(t) \leq 0$ on $[a, b]$, then we say that $u$ is concave down on $[a, b]$.

By a function $u(t)$ being a positive solution of (3)-(4), we mean that $u(t)$ is positive on $(a, b)$ and satisfies (3)-(4). Let $G(t, s)$ be Green's function of $u^{\Delta \Delta}(t)=0$ with respect to boundary condition (4); then

$$
\begin{aligned}
& G(t, s) \\
& = \begin{cases}\frac{1}{d}(\gamma b+\delta-\gamma t)(\beta+\alpha(\rho(s)-\rho(a))): & \rho(s) \leq t, \\
\frac{1}{d}(\gamma b+\delta-\gamma \rho(s))(\beta+\alpha(t-\rho(a))): & t \leq \rho(s),\end{cases}
\end{aligned}
$$

where $\alpha, \beta, \gamma, \delta \geq 0: d=\gamma \beta+\alpha \delta+\alpha \gamma(b-\rho(a))>0$.

This implies that $G(t, s)$ satisfies

$$
\begin{aligned}
0 \leq G(t, s) \leq G(\rho(s), s) & \\
& \text { for } t \in[\rho(a), b], s \in[a, b] .
\end{aligned}
$$

In fact, on the one hand, from Green's function, if $s$ is kept fixed then $G(t, s)$ is a decreasing function of $t$ for $t>\rho(s)$ and increasing for $t<\rho(s)$. This leads to the conclusion that $G(t, s)$ has a global maximum at $t=\rho(s)$, so $G(t, s) \leq$ $G(\rho(s), s)$, for $t \in[\rho(a), b]$. On the other hand, we can easily know that $G(t, s)$ is nonnegative from the following two equalities:

$$
\begin{gathered}
\gamma b+\delta-\gamma t=\delta+\gamma(b-t), \\
\gamma b+\delta-\gamma \rho(s)=\delta+\gamma(b-\rho(s)) .
\end{gathered}
$$

Let the Banach space $X=C^{2}[\rho(a), b]$ be equipped with the norm

$$
\begin{aligned}
& \|u\|=\max \left\{\max _{t \in[\rho(a), b]}|u(t)|, \max _{t \in[\rho(a), b]}\left|u^{\Delta}(t)\right|,\right. \\
& \left.\max _{t \in[\rho(a), b]}\left|u^{\Delta \Delta}(t)\right|\right\}, \\
& C^{2+}[\rho(a), b]=\left\{u \in C^{2}[\rho(a), b]: u(t) \geq 0, t\right. \\
& \in[\rho(a), b]\} .
\end{aligned}
$$

Definition 6. Let $X$ be a real Banach space. Let $P$ be a nonempty, convex closed set in $X$. We say that $P$ is a cone it it satisfies the following properties: (i) $\lambda u \in P$ for $u \in P, \lambda \geq 0$;

(ii) $u,-u \in P$ implies $u=\theta$ ( $\theta$ denotes the null element of $X)$.

Define the cone $P \subset X$ by

$$
P=\left\{u \in C^{2+}[\rho(a), b]: u(t)\right.
$$

$$
\text { is a concave function on }[\rho(a), b]\} \text {. }
$$

We define operator $A: C^{2+}[\rho(a), b] \rightarrow C[\rho(a), b]$ by

$$
\begin{array}{r}
(A u)(t)=\int_{\rho(a)}^{b} G(t, s) \\
\cdot \phi_{q}\left(\int_{\rho(a)}^{s} q(\tau) f\left(\tau, u(\tau), u^{\Delta \Delta}(\tau)\right) \nabla \tau\right) \Delta s, \\
\forall u \in C^{2+}[\rho(a), b],
\end{array}
$$

and then all the fixed points of operator $A$ are the solutions for BVP (3)-(4).

Remark 7. From the definition of the operator $A$, we can easily get the following properties:

$$
\begin{aligned}
& (A u)^{\Delta}(t)=-\frac{\gamma}{d} \int_{\rho(a)}^{t}(\beta+\alpha(\rho(s)-\rho(a))) \\
& \cdot \phi_{q}\left(\int_{\rho(a)}^{s} q(\tau) f\left(\tau, u(\tau), u^{\Delta \Delta}(\tau)\right) \nabla \tau\right) \Delta s+\frac{\alpha}{d} \\
& \cdot \int_{t}^{b}(\gamma b+\delta-\gamma \rho(s)) \\
& \cdot \phi_{q}\left(\int_{\rho(a)}^{s} q(\tau) f\left(\tau, u(\tau), u^{\Delta \Delta}(\tau)\right) \nabla \tau\right) \Delta s, \\
& (A u)^{\Delta \Delta}(t) \\
& =-\phi_{q}\left(\int_{\rho(a)}^{t} q(\tau) f\left(\tau, u(\tau), u^{\Delta \Delta}(\tau)\right) \nabla \tau\right) \Delta s .
\end{aligned}
$$

Lemma 8. Assume that (H1)-(H3) hold; then the operator A : $P \rightarrow P$ is completely continuous.

Proof. From (H1)-(H3) and the definition of $A$, we deduce that, for any $u \in P$, there is $(A u)(t) \geq 0$. By (24), we get

$$
\begin{aligned}
& (A u)^{\Delta \Delta}(t) \\
& \quad=-\phi_{q}\left(\int_{\rho(a)}^{t} q(\tau) f\left(\tau, u(\tau), u^{\Delta \Delta}(\tau)\right) \nabla \tau\right) \Delta s
\end{aligned}
$$

which implies that $(A u)$ is concave on $[\rho(a), b]$. Thus, we have $A(P) \subset P$.

Now we show that $A: P \rightarrow P$ is completely continuous. The continuity of $A$ is obvious. We prove $A$ is compact. Let $\Omega_{0} \subset P$ be a bounded set. Then, there exists $R$ such that $\Omega_{0} \subset$ $\{u \in P:\|u\| \leq R\}$. We can easily prove that $A\left(\Omega_{0}\right)$ is bounded 
and equicontinuous; that is, $A\left(\Omega_{0}\right)$ is a relatively compact set according to the Ascoli-Arzela theorem. Thus $A: P \rightarrow P$ is completely continuous.

For convenience we use the following notations. Let

$$
\begin{aligned}
B & =\max \left\{\int_{\rho(a)}^{b} G(\rho(s), s)=\frac{1}{d}\left[\frac{\beta \gamma}{2}\left((\rho(a))^{2}+b^{2}\right)\right.\right. \\
& +\beta \delta(b-\rho(a))+\frac{\alpha \delta}{2}(b-\rho(a))^{2} \\
& \left.+\frac{\alpha \gamma}{6}\left(2(\rho(a))^{3}+b^{3}-3(\rho(a))^{2}+3(\rho(a))^{2} b-3 \rho(a) b^{2}\right)\right], \\
& \frac{\alpha}{d}\left(\frac{1}{2} \gamma(b-\rho(a))^{2}+\delta(b-\rho(a))\right), \frac{\gamma}{d}\left(\frac{1}{2} \alpha(b-\rho(a))^{2}\right. \\
& +\beta(b-\rho(a))), 1\}, \\
C & =B \phi_{q}\left(\int_{\rho(a)}^{b} q(\tau) \nabla \tau\right) .
\end{aligned}
$$

\section{The Main Results}

We now give our results for the existence of positive solutions for BVP (3) and (4).

Theorem 9. Assume that (H1)-(H4) hold and there exists a positive constant $l>1$ such that

(H5) $f\left(t, x_{1}, y_{1}\right) \leq f\left(t, x_{2}, y_{2}\right)$ for any $\rho(a) \leq t \leq b, 0 \leq$ $x_{1} \leq x_{2} \leq l, 0 \leq\left|y_{1}\right| \leq\left|y_{2}\right| \leq l$

(H6) $\phi_{p}(C) \leq \phi_{p}(l) / k l^{\mu_{1}+\mu_{2}}$,

where $\mu_{1}, \mu_{2}, k$ are defined by (5), (6), and (7), respectively. Then boundary value (3) and (4) has two positive, concave solutions $u^{*}$ and $v^{*}$ such that

$$
\begin{aligned}
0 & <\left\|u^{*}\right\| \leq l, \\
\lim _{n \rightarrow \infty} u_{n} & =\lim _{n \rightarrow \infty} A^{n} u_{0}=u^{*}, \\
\lim _{n \rightarrow \infty}\left(u_{n}\right)^{\Delta \Delta} & =\lim _{n \rightarrow \infty}\left(A^{n} u_{0}\right)^{\Delta \Delta}=\left(u^{*}\right)^{\Delta \Delta},
\end{aligned}
$$

where

$$
\begin{aligned}
u_{0}(t) & =\frac{l}{B} \int_{\rho(a)}^{b} G(t, s) \Delta s, \quad \rho(a) \leq t \leq b, \\
0 & <\left\|v^{*}\right\| \leq l, \\
\lim _{n \rightarrow \infty} v_{n} & =\lim _{n \rightarrow \infty} A^{n} v_{0}=v^{*}, \\
\lim _{n \rightarrow \infty}\left(v_{n}\right)^{\Delta \Delta} & =\lim _{n \rightarrow \infty}\left(A^{n} v_{0}\right)^{\Delta \Delta}=\left(v^{*}\right)^{\Delta \Delta},
\end{aligned}
$$

where $v_{0}(t)=0, \rho(a) \leq t \leq b, A$ is defined by (22). The iterative schemes in this theorem are $u_{0}(t), u_{n+1}=A u_{n}=A^{n} u_{0}$, $n=0,1,2, \ldots$ and $v_{0}(t), v_{n+1}=A v_{n}=A^{n} v_{0}, n=0,1,2, \ldots$
Proof. We define $\bar{P}_{l}=\{u \in P:\|u\| \leq l\}$. Then, in what follows, we will prove that $T: \bar{P}_{l} \rightarrow \bar{P}_{l}$. If $u \in \bar{P}_{l}$, then $\|u\| \leq l$; we have

$$
\begin{aligned}
0 & \leq u(t) \leq \max _{t \in[\rho(a), b]}|u(t)| \leq\|u\| \leq l, \\
\left|u^{\Delta \Delta}(t)\right| & \leq \max _{t \in[\rho(a), b]}\left|u^{\Delta \Delta}(t)\right| \leq\|u\| \leq l .
\end{aligned}
$$

So by (8), (9) and (H5), we have

$$
\begin{aligned}
0 & \leq f\left(t, u(t), u^{\Delta \Delta}(t)\right) \leq f(t, l, l) \leq l^{\mu_{1}+\mu_{2}} f(t, 1,1) \\
& \leq k l^{\mu_{1}+\mu_{2}}, \quad \text { for } \rho(a)<t<b .
\end{aligned}
$$

In fact,

$$
\begin{gathered}
\|A u\|=\max \left\{\max _{t \in[\rho(a), b]}|(A u)(t)|, \max _{t \in[\rho(a), b]}\left|(A u)^{\Delta}(t)\right|,\right. \\
\left.\max _{t \in[\rho(a), b]}\left|(A u)^{\Delta \Delta}(t)\right|\right\}=\max \left\{\max _{t \in[\rho(a), b]}|(A u)(t)|,\right. \\
\left.(A u)^{\Delta}(\rho(a)),-(A u)^{\Delta}(b),-(A u)^{\Delta \Delta}(b)\right\} .
\end{gathered}
$$

Then, by (18), (30) and (H6), we have

$$
\begin{aligned}
& \max _{t \in[\rho(a), b]}|(A u)(t)|=\max _{t \in[\rho(a), b]} \mid \int_{\rho(a)}^{b} G(t, s) \\
& \cdot \phi_{q}\left(\int_{\rho(a)}^{s} q(\tau) f\left(\tau, u(\tau), u^{\Delta \Delta}(\tau)\right) \nabla \tau\right) \Delta s \mid \\
& \leq \int_{\rho(a)}^{b} G(\rho(s), s) \\
& \cdot \phi_{q}\left(\int_{\rho(a)}^{s} q(\tau) f\left(\tau, u(\tau), u^{\Delta \Delta}(\tau)\right) \nabla \tau\right) \Delta s \\
& \leq \phi_{q}\left(\int_{\rho(a)}^{s} q(\tau) f\left(\tau, u(\tau), u^{\Delta \Delta}(\tau)\right) \nabla \tau\right) \\
& \cdot \int_{\rho(a)}^{b} G(\rho(s), s) \Delta s \leq \phi_{q}\left(k l^{\mu_{1}+\mu_{2}}\right) \\
& \cdot \phi_{q}\left(\int_{\rho(a)}^{b} q(\tau) \nabla \tau\right) \int_{\rho(a)}^{b} G(\rho(s), s) \Delta s \\
& \leq C \phi_{q}\left(k l^{\mu_{1}+\mu_{2}}\right) \leq l,
\end{aligned}
$$


International Journal of Differential Equations

5

and from (23) and (24), we have

$$
\begin{aligned}
& (A u)^{\Delta}(\rho(a))=\frac{\alpha}{d} \int_{\rho(a)}^{b}(\gamma b+\delta-\gamma \rho(s)) \\
& \cdot \phi_{q}\left(\int_{\rho(a)}^{s} q(\tau) f\left(\tau, u(\tau), u^{\Delta \Delta}(\tau)\right) \nabla \tau\right) \Delta s \leq \frac{\alpha}{d} \\
& \cdot \int_{\rho(a)}^{b}(\gamma b+\delta-\gamma \rho(s)) \\
& \cdot \phi_{q}\left(\int_{\rho(a)}^{b} q(\tau) f\left(\tau, u(\tau), u^{\Delta \Delta}(\tau)\right) \nabla \tau\right) \Delta s \leq \frac{\alpha}{d} \\
& \cdot \phi_{q}\left(k l^{\mu_{1}+\mu_{2}}\right) \phi_{q}\left(\int_{\rho(a)}^{b} q(\tau) \nabla \tau\right) \\
& \cdot \int_{\rho(a)}^{b}(\gamma b+\delta-\gamma \rho(s)) \Delta s \\
& =\frac{\alpha}{d}\left(\frac{1}{2} \gamma(b-\rho(a))^{2}+\delta(b-\rho(a))\right) \phi_{q}\left(k l^{\mu_{1}+\mu_{2}}\right) \\
& \cdot \phi_{q}\left(\int_{\rho(a)}^{b} q(\tau) \nabla \tau\right) \leq C \phi_{q}\left(k l^{\mu_{1}+\mu_{2}}\right) \leq l, \\
& -(A u)^{\Delta}(b)=\frac{\gamma}{d} \int_{\rho(a)}^{b}(\beta+\alpha(\rho(s)-\rho(a))) \\
& \cdot \phi_{q}\left(\int_{\rho(a)}^{s} q(\tau) f\left(\tau, u(\tau), u^{\Delta \Delta}(\tau)\right) \nabla \tau\right) \Delta s \leq \frac{\gamma}{d} \\
& \cdot \int_{\rho(a)}^{b}(\beta+\alpha(\rho(s)-\rho(a))) \\
& \cdot \phi_{q}\left(\int_{\rho(a)}^{b} q(\tau) f\left(\tau, u(\tau), u^{\Delta \Delta}(\tau)\right) \nabla \tau\right) \Delta s \leq \frac{\gamma}{\rho} \\
& \cdot \phi_{q}\left(k l^{\mu_{1}+\mu_{2}}\right) \phi_{q}\left(\int_{\rho(a)}^{b} q(\tau) \nabla \tau\right) \\
& \cdot \int_{\rho(a)}^{b}(\beta+\alpha(\rho(s)-\rho(a))) \Delta s \\
& =\frac{\gamma}{d}\left(\frac{1}{2} \alpha(b-\rho(a))^{2}+\beta(b-\rho(a))\right) \phi_{q}\left(k l^{\mu_{1}+\mu_{2}}\right) \\
& \cdot \phi_{q}\left(\int_{\rho(a)}^{b} q(\tau) \nabla \tau\right) \leq C \phi_{q}\left(k l^{\mu_{1}+\mu_{2}}\right) \leq l, \\
& -(A u)^{\Delta \Delta}(b) \\
& =\phi_{q}\left(\int_{\rho(a)}^{b} q(\tau) f\left(\tau, u(\tau), u^{\Delta \Delta}(\tau)\right) \nabla \tau\right) \\
& \leq \phi_{q}\left(k l^{\mu_{1}+\mu_{2}}\right) \phi_{q}\left(\int_{\rho(a)}^{b} q(\tau) \nabla \tau\right) \leq C \phi_{q}\left(k l^{\mu_{1}+\mu_{2}}\right) \\
& \leq l \text {. }
\end{aligned}
$$

Thus, we obtained that $\|A u\| \leq l$. So, we have shown that $T \bar{P}_{l} \subset \bar{P}_{l}$.

Let $u_{0}(t)=(l / B) \cdot \int_{\rho(a)}^{b} G(t, s) \Delta s, t \in[\rho(a), b]$, then

$$
\begin{aligned}
& u_{0}^{\Delta}(t)=\frac{l}{B}\left[-\frac{\gamma}{d} \int_{\rho(a)}^{t}(\beta+\alpha(\rho(s)-\rho(a))) \Delta s\right. \\
& \left.+\frac{\alpha}{d} \int_{t}^{b}(\gamma b+\delta-\gamma \rho(s)) \Delta s\right], \\
& u_{0}^{\Delta \Delta}(t)=-\frac{l}{B} .
\end{aligned}
$$

It is easy to get

$$
\begin{aligned}
& u_{0}(t) \leq \frac{l}{\int_{\rho(a)}^{b} G(\rho(s), s) \Delta s} \int_{\rho(a)}^{b} G(t, s) \Delta s \leq l, \\
& u_{0}^{\Delta}(\rho(a))=\frac{l}{B} \cdot \frac{\alpha}{d}\left(\frac{1}{2} \gamma(b-\rho(a))^{2}+\delta(b-\rho(a))\right) \\
& \leq l \text {, } \\
& -u_{0}^{\Delta}(b)=\frac{l}{B} \cdot \frac{\gamma}{d}\left(\frac{1}{2} \alpha(b-\rho(a))^{2}+\beta(b-\rho(a))\right) \\
& \leq l \text {, } \\
& \max _{t \in[\rho(a), b]}\left|u_{0}^{\Delta \Delta}(t)\right|=\frac{l}{B} \leq l,
\end{aligned}
$$

Let $u_{1}=A u_{0}$, then from the above expression, we have $u_{1} \in \bar{P}_{l}$. We define

$$
u_{n+1}=A u_{n}=A^{n+1} u_{0}, \quad n=0,1,2, \ldots
$$

It follows from $A \bar{P}_{l} \subset \bar{P}_{l}$, that $u_{n} \in \bar{P}_{l}, n=1,2, \ldots$. Since $A$ is completely continuous, we can assert that $\left\{u_{n}\right\}$ is a sequentially compact set. Since

$$
\begin{aligned}
& u_{1}(t)=\left(A u_{0}\right)(t)=\int_{\rho(a)}^{b} G(t, s) \\
& \cdot \phi_{q}\left(\int_{\rho(a)}^{s} q(\tau) f\left(\tau, u_{0}(\tau), u_{0}^{\Delta \Delta}(\tau)\right) \nabla \tau\right) \Delta s \\
& \leq \int_{\rho(a)}^{b} G(t, s) \phi_{q} \\
& \cdot\left(\int_{\rho(a)}^{b} q(\tau) f\left(\tau, u_{0}(\tau), u_{0}^{\Delta \Delta}(\tau)\right) \nabla \tau\right) \Delta s \\
& \leq \phi_{q}\left(k l^{\mu_{1}+\mu_{2}}\right) \phi_{q}\left(\int_{\rho(a)}^{b} q(\tau) \nabla \tau\right) \int_{\rho(a)}^{b} G(t, s) \Delta s \\
& =\frac{C}{B} \phi_{q}\left(k l^{\mu_{1}+\mu_{2}}\right) \int_{\rho(a)}^{b} G(t, s) \Delta s \leq \frac{l}{B} \\
& \cdot \int_{\rho(a)}^{b} G(t, s) \Delta s=u_{0}(t),
\end{aligned}
$$




$$
\begin{aligned}
& \left|u_{1}^{\Delta \Delta}(t)\right|=\left|\left(A u_{0}\right)^{\Delta \Delta}(t)\right| \\
& \quad=\phi_{q}\left(\int_{\rho(a)}^{t} q(\tau) f\left(\tau, u_{0}(\tau), u_{0}^{\Delta \Delta}(\tau)\right) \nabla \tau\right) \\
& \quad \leq \phi_{q}\left(\int_{\rho(a)}^{b} q(\tau) f\left(\tau, u_{0}(\tau), u_{0}^{\Delta \Delta}(\tau)\right) \nabla \tau\right) \\
& \quad \leq \phi_{q}\left(k l^{\mu_{1}+\mu_{2}}\right) \phi_{q}\left(\int_{\rho(a)}^{b} q(\tau) \nabla \tau\right)=\frac{C}{B} \phi_{q}\left(k l^{\mu_{1}+\mu_{2}}\right) \\
& \quad \leq \frac{l}{B}=\left|u_{0}^{\Delta \Delta}(t)\right|
\end{aligned}
$$

we obtain

$$
\begin{gathered}
u_{1}(t) \leq u_{0}(t), \\
\left|u_{1}^{\Delta \Delta}(t)\right| \leq\left|u_{0}^{\Delta \Delta}(t)\right|, \\
\rho(a) \leq t \leq b .
\end{gathered}
$$

By (22) and (H5), we have

$$
\begin{aligned}
u_{2}(t) & =A u_{1}(t) \leq A u_{0}(t)=u_{1}(t), \\
\rho(a) & \leq t \leq b, \\
\left|u_{2}^{\Delta \Delta}(t)\right| & =\left|\left(A u_{1}\right)^{\Delta \Delta}(t)\right| \leq\left|\left(A u_{0}\right)^{\Delta \Delta}(t)\right|=\left|u_{1}^{\Delta \Delta}(t)\right|, \\
\rho(a) & \leq t \leq b .
\end{aligned}
$$

Hence by induction we have

$$
\begin{gathered}
u_{n+1}(t) \leq u_{n}(t), \\
\left|u_{n+1}^{\Delta \Delta}(t)\right| \leq\left|u_{n}^{\Delta \Delta}(t)\right|, \\
\rho(a) \leq t \leq b,
\end{gathered}
$$

$$
n=0,1,2, \ldots
$$

Thus, there exists $u^{*} \in \bar{P}_{l}$ such that $u_{n} \rightarrow u^{*}$. Applying the continuity of $A$ and $u_{n+1}(t)=A u_{n}(t)$, we get $A u^{*}(t)=u^{*}(t)$ which implies that $u^{*}$ is a nonnegative concave solution of the BVP (3) and (4).

On the other hand, let $v_{0}(t)=0, t \in[\rho(a), b]$, then $v_{0}(t) \in \bar{P}_{l}$. Let $v_{1}=A v_{0}=A 0$, then $v_{1} \in \bar{P}_{l}$. We define $v_{n+1}=A v_{n}=A^{n+1} v_{0}, n=0,1,2, \ldots$ Since $A: \bar{P}_{l} \rightarrow \bar{P}_{l}$, we have $v_{n} \in A \bar{P}_{l} \subseteq \bar{P}_{l}, n=1,2, \ldots$. Since $A$ is completely continuous, we can assert that $\left\{v_{n}\right\}$ is a sequentially compact set.

Since $v_{1}=A v_{0}=A 0 \in \bar{P}_{l}$, we have

$$
\begin{aligned}
v_{1}(t) & =\left(A v_{0}\right)(t)=(A 0)(t) \geq 0, \\
\rho(a) & \leq t \leq b, \\
\left|v_{1}^{\Delta \Delta}(t)\right| & =\left|\left(A v_{0}\right)^{\Delta \Delta}(t)\right|=\left|(A 0)^{\Delta \Delta}(t)\right| \geq 0, \\
\rho(a) & \leq t \leq b .
\end{aligned}
$$

So,

$$
\begin{aligned}
v_{2}(t) & =\left(A v_{1}\right)(t) \geq(A 0)(t)=v_{1}(t), \\
\rho(a) & \leq t \leq b, \\
\left|v_{2}^{\Delta \Delta}(t)\right| & =\left|\left(A v_{1}\right)^{\Delta \Delta}(t)\right| \geq\left|(A 0)^{\Delta \Delta}(t)\right|=\left|v_{1}^{\Delta \Delta}(t)\right|, \\
\rho(a) & \leq t \leq b .
\end{aligned}
$$

By an induction argument similar to the above we obtain

$$
\begin{gathered}
v_{n+1}(t) \geq v_{n}(t), \\
\left|v_{n+1}^{\Delta \Delta}(t)\right| \geq\left|v_{n}^{\Delta \Delta}(t)\right|, \\
\rho(a) \leq t \leq b,
\end{gathered}
$$

$$
n=0,1,2, \ldots
$$

Hence, there exists $v^{*} \in \bar{P}_{l}$ such that $v_{n} \rightarrow v^{*}$. Applying the continuity of $A$ and $v_{n+1}(t)=A v_{n}(t)$, we obtain $A v^{*}(t)=$ $v^{*}(t)$ which implies that $v^{*}$ is a nonnegative solution of the BVP (3) and (4). From the condition (H4), we know that the zero function is not the solution of the BVP (3) and (4). Thus, $\max _{\rho(a) \leq t \leq b}\left|v^{*}(t)\right|>0$, and by the concavity of $v^{*}(t)$ we have

$$
\begin{array}{r}
v^{*}(t) \geq \min \{t-\rho(a), b-t\} \max _{t \in[\rho(a), b]} v^{*}(t)>0, \\
\quad \rho(a)<t<b .
\end{array}
$$

Next, noting $u_{0}(t) \geq v_{0}(t),\left|u_{0}^{\Delta \Delta}(t)\right| \geq\left|v_{0}^{\Delta \Delta}(t)\right|, t \in$ $[\rho(a), b]$, it follows from (H5) and (22) that $A u_{0} \geq A v_{0}$, and by induction, we have $u_{n}(t) \geq v_{n}(t), n=0,1,2, \ldots$ which implies that $u^{*} \geq v^{*}$. Thus we have

$$
\begin{aligned}
u^{*}(t) & \geq v^{*}(t) \geq \min \{t-\rho(a), b-t\} \max _{t \in[\rho(a), b]} v^{*}(t) \\
& >0 .
\end{aligned}
$$

This means that $u^{*}$ is a positive concave solution of the BVP (3) and (4). Thus the BVP (3) and (4) has two positive and concave solutions $u^{*}, v^{*}$ such that $0 \leq\left\|u^{*}\right\| \leq l, 0 \leq$ $\left\|v^{*}\right\| \leq l$, and, from the above proof, we know that the iterative sequences hold. The proof is completed.

\section{Example}

Now, we present an example to illustrate the main result: Theorem 9. 
Let $\mathbb{T}=\left\{(2 / 5)^{n}: n \in \mathbb{N}_{0}\right\} \cup\{0\} \cup[1,2]$. We consider the following third-order Sturm-Liouville boundary value problem with $p$-Laplacian on time scales:

$$
\begin{aligned}
& \left(\phi_{3}\left(u^{\Delta \Delta}(t)\right)\right)^{\nabla} \\
& +\frac{1}{\sqrt{t}}\left[e^{t}\left(u^{3 / 5}(t)+\left(u^{\Delta \Delta}\right)^{1 / 3}(t)\right)+1\right]=0, \\
& t \in\left[\frac{4}{25}, \frac{2}{5}\right], \\
& u\left(\frac{8}{125}\right)-u^{\Delta}\left(\frac{8}{125}\right)=0, \\
& u\left(\frac{2}{5}\right)=0, \\
& u^{\Delta \Delta}\left(\frac{8}{125}\right)=0,
\end{aligned}
$$

where $p=3, \alpha=1, \beta=1, \gamma=1$, and $\delta=0$. Let

$$
\begin{aligned}
f(t, u, v) & =e^{t}\left(u^{3 / 5}+v^{1 / 3}\right)+1, \\
q(t) & =\frac{1}{\sqrt{t}}, \\
\mu_{1} & =\frac{1}{2}, \\
\mu_{2} & =\frac{1}{3},
\end{aligned}
$$

where, for any $0 \leq \lambda \leq 1$ and $u \in[0, \infty), v \in \mathbb{R}, t \in$ $[8 / 125,2 / 5]$, we have

$$
\begin{aligned}
f(t, \lambda u, v) & =e^{t}\left[(\lambda u)^{3 / 5}+v^{1 / 3}\right]+1 \\
& \geq \lambda^{3 / 5}\left[e^{t}\left(u^{3 / 5}+v^{1 / 3}\right)+1\right] \\
& \geq \lambda^{1 / 2} f(t, u, v), \\
f(t, u, \lambda v) & =e^{t}\left[u^{3 / 5}+(\lambda v)^{1 / 3}\right]+1 \\
& \geq \lambda^{1 / 3}\left[e^{t}\left(u^{3 / 5}+v^{1 / 3}\right)+1\right] \\
& \geq \lambda^{1 / 3} f(t, u, v),
\end{aligned}
$$

which implies that (H3) holds.

On the other hand, it is clear that (H1), (H2), and (H4) are satisfied and

$$
\begin{aligned}
& d=\frac{167}{125}, \\
& k=\max _{t \in[8 / 125,2 / 5]} f(t, 1,1)=2 e^{2 / 5}+1 .
\end{aligned}
$$

Since $q(t)=1 / \sqrt{t}$, we have

$$
\begin{aligned}
& B=\max \{0.06,0.0425,0.2937,1\}=1, \\
& C=B \phi_{q}\left(\int_{8 / 125}^{2 / 5} q(\tau) \nabla \tau\right)=\phi_{q}(0.759),
\end{aligned}
$$

so $\phi_{p}(C)=0.759 \phi_{p}(1)=0.759$.

$$
\text { Take } l=\left[\left(2 e^{2 / 5}+1\right)\right]^{6 / 7} ; \text { then }
$$

$$
\begin{aligned}
\frac{\phi_{p}(l)}{k l^{\mu_{1}+\mu_{2}}} & =\frac{\left(\left(2 e^{2 / 5}+1\right)^{6 / 7}\right)^{2}}{\left(2 e^{2 / 5}+1\right)\left(\left[\left(2 e^{2 / 5}+1\right)\right]^{6 / 7}\right)^{5 / 6}}=1 \\
> & 0.759=\phi_{p}(C),
\end{aligned}
$$

which implies that (H6) holds. For $l=\left[\left(2 e^{2 / 5}+1\right)\right]^{6 / 7}$, it is clear that (H5) holds. So, by Theorem 9, boundary value problem (46) has two positive and concave solutions $u^{*}$ and $v^{*}$ such that

$$
\begin{aligned}
0 & <u^{*}<\left[\left(2 e^{2 / 5}+1\right)\right]^{6 / 7}, \\
0 & <\left|\left(u^{*}\right)^{\Delta}\right|<\left[\left(2 e^{2 / 5}+1\right)\right]^{6 / 7}, \\
0 & <\left|\left(u^{*}\right)^{\Delta \Delta}\right|<\left[\left(2 e^{2 / 5}+1\right)\right]^{6 / 7}, \\
\lim _{n \rightarrow \infty} u_{n} & =\lim _{n \rightarrow \infty} A^{n} u_{0}=u^{*}, \\
\lim _{n \rightarrow \infty}\left(u_{n}\right)^{\Delta \Delta} & =\lim _{n \rightarrow \infty}\left(A^{n} u_{0}\right)^{\Delta \Delta}=\left(u^{*}\right)^{\Delta \Delta},
\end{aligned}
$$

where $u_{0}(t)=-1.0688 t^{2}+0.0666 t+0.00308, t \in[8 / 125$, $2 / 5]$, and

$$
\begin{aligned}
0 & <v^{*}<\left[\left(2 e^{2 / 5}+1\right)\right]^{6 / 7}, \\
0 & <\left|\left(v^{*}\right)^{\Delta}\right|<\left[\left(2 e^{2 / 5}+1\right)\right]^{6 / 7}, \\
0 & <\left|\left(v^{*}\right)^{\Delta \Delta}\right|<\left[\left(2 e^{2 / 5}+1\right)\right]^{6 / 7}, \\
\lim _{n \rightarrow \infty} v_{n} & =\lim _{n \rightarrow \infty} A^{n} v_{0}=v^{*}, \\
\lim _{n \rightarrow \infty}\left(v_{n}\right)^{\Delta \Delta} & =\lim _{n \rightarrow \infty}\left(A^{n} v_{0}\right)^{\Delta \Delta}=\left(v^{*}\right)^{\Delta \Delta},
\end{aligned}
$$

where $v_{0}(t)=0, t \in[8 / 125,2 / 5]$.

\section{Conflict of Interests}

The author declares that there is no conflict of interests regarding the publication of this paper.

\section{References}

[1] R. P. Agarwal, M. Bohner, and P. Rehak, "Half-linear dynamic equations," in Nonlinear Analysis and Applications: to V. Lakshmikantham on his 80th Birthday, pp. 1-57, Kluwer Academic Publishers, Dordrecht,, 2003. 
[2] R. P. Agarwal and D. O’Regan, "Nonlinear boundary value problems on time scales," Nonlinear Analysis: Theory, Methods \& Applications, vol. 44, no. 4, pp. 527-535, 2001.

[3] D. R. Anderson, R. Avery, and J. Henderson, "Existence of solutions for a one dimensional $p$-Laplacian on time-scales," Journal of Difference Equations and Applications, vol. 10, no. 10, pp. 889-896, 2004.

[4] D. R. Anderson, G. S. Guseinov, and J. Hoffacker, "Higher-order selfadjoint boundary-value problems on time scales," Journal of Computational and Applied Mathematics, vol. 194, no. 2, pp. 309-342, 2006.

[5] F. M. Atici and G. Sh. Guseinov, "On Green's functions and positive solutions for boundary value problems on time scales," Journal of Computational and Applied Mathematics, vol. 141, no. 1-2, pp. 75-99, 2002.

[6] L. H. Erbe and A. Peterson, "Positive solutions for a nonlinear differential equation on a measure chain," Mathematical and Computer Modelling, vol. 32, no. 5-6, pp. 571-585, 2000.

[7] X. Feng, H. Feng, and H. Tan, "Existence and iteration of positive solutions for third-order Sturm-Liouville boundary value problems with p-Laplacian," Applied Mathematics and Computation, vol. 266, pp. 634-641, 2015.

[8] W. Han and S. Kang, "Multiple positive solutions of nonlinear third-order BVP for a class of $p$-Laplacian dynamic equations on time scales," Mathematical and Computer Modelling, vol. 49, no. 3-4, pp. 527-535, 2009.

[9] Z. He and X. Jiang, "Triple positive solutions of boundary value problems for $p$-Laplacian dynamic equations on time scales," Journal of Mathematical Analysis and Applications, vol. 321, no. 2, pp. 911-920, 2006.

[10] H. Su, B. Wang, and Z. Wei, "Positive solutions of four-point boundary value problems for four-order $p$-Laplacian dynamic equations on time scales," Electronic Journal of Differential Equations, vol. 78, pp. 1-13, 2006.

[11] H.-R. Sun and W.-T. Li, "Multiple positive solutions for $p$ Laplacian $m$-point boundary value problems on time scales," Applied Mathematics and Computation, vol. 182, no. 1, pp. 478491, 2006.

[12] S. Hilger, "Analysis on measure chains-a unified approach to continuous and discrete calculus," Results in Mathematics, vol. 18, no. 1-2, pp. 18-56, 1990.

[13] M. Bohner and A. Peterson, Dynamic Equations on Time Scales: An Introduction with Applications, Birkhäuser, Boston, Mass, USA, 2001.

[14] M. Bohner and A. Peterson, Advances in Dynamic Equations on Time Scales, Birkhäauser, Boston, Mass, USA, 2003.

[15] Z.-C. Hao, J. Liang, and T.-J. Xiao, "Existence results for time scale boundary value problem," Journal of Computational and Applied Mathematics, vol. 197, no. 1, pp. 156-168, 2006.

[16] R. Y. Ma and N. Castaneda, "Existence of solutions of nonlinear $m$-point boundary value problem," Journal of Mathematical Analysis and Applications, vol. 256, no. 2, pp. 556-567, 2001.

[17] M. R. Grossinho, F. M. Minhós, and A. I. Santos, "Existence result for a third-order ODE with nonlinear boundary conditions in presence of a sign-type Nagumo control," Journal of Mathematical Analysis and Applications, vol. 309, no. 1, pp. 271283, 2005.

[18] S. H. Li, "Positive solutions of nonlinear singular third-order two-point boundary value problem," Journal of Mathematical Analysis and Applications, vol. 323, no. 1, pp. 413-425, 2006.
[19] D.-X. Ma, Z.-J. Du, and W.-G. Ge, "Existence and iteration of monotone positive solutions for multipoint boundary value problem with $p$-Laplacian operator," Computers \& Mathematics with Applications, vol. 50, no. 5-6, pp. 729-739, 2005.

[20] H. Su, Z. Wei, and F. Xu, “The existence of countably many positive solutions for a system of nonlinear singular boundary value problems with the $p$-Laplacian operator," Journal of Mathematical Analysis and Applications, vol. 325, no. 1, pp. 319-332, 2007.

[21] H.-R. Sun and W.-T. Li, "Existence theory for positive solutions to one-dimensional $p$-Laplacian boundary value problems on time scales," Journal of Differential Equations, vol. 240, no. 2, pp. 217-248, 2007.

[22] C. Yang and J. Yan, "Positive soluitons for third-order SturmLiouville boundary value problems with $p$-Laplacian," Computers \& Mathematics with Applications, vol. 59, no. 6, pp. 20592066, 2010.

[23] C. Z. Bai and J. X. Fang, "Existence of multiple positive solutions for nonlinear $m$-point boundary value problems," Journal of Mathematical Analysis and Applications, vol. 281, no. 1, pp. 7685, 2003. 


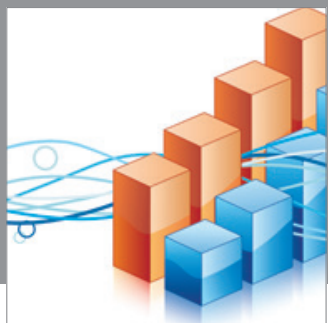

Advances in

Operations Research

mansans

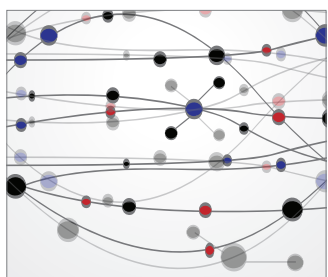

The Scientific World Journal
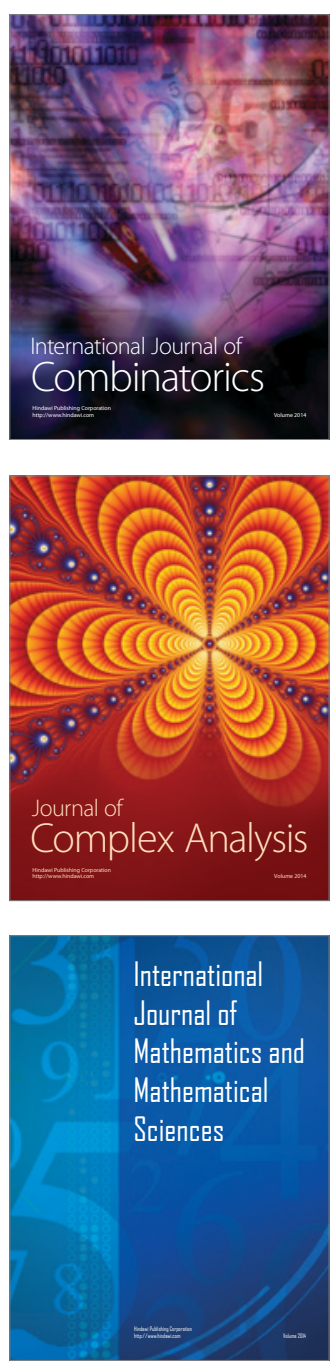
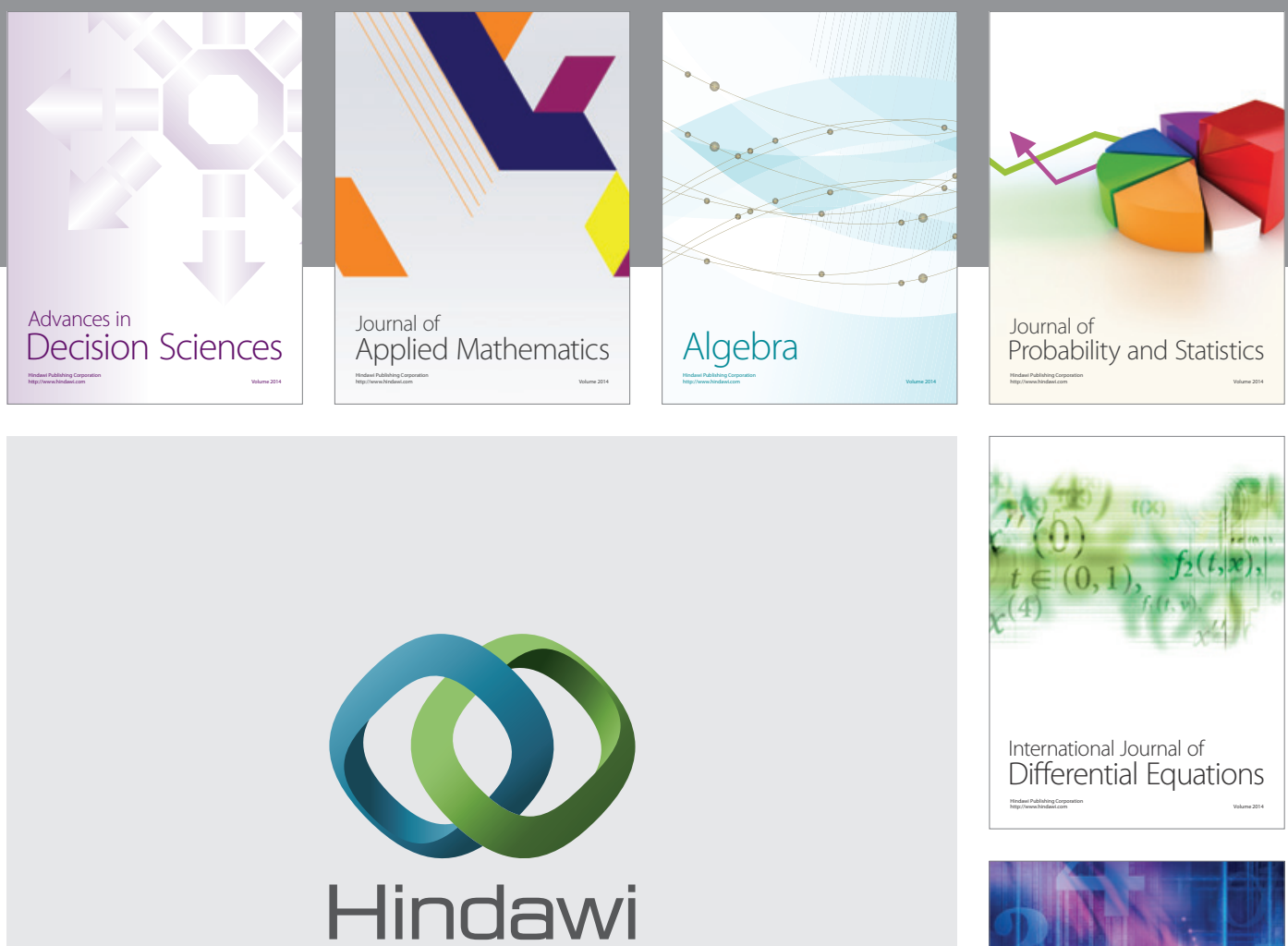

Submit your manuscripts at http://www.hindawi.com
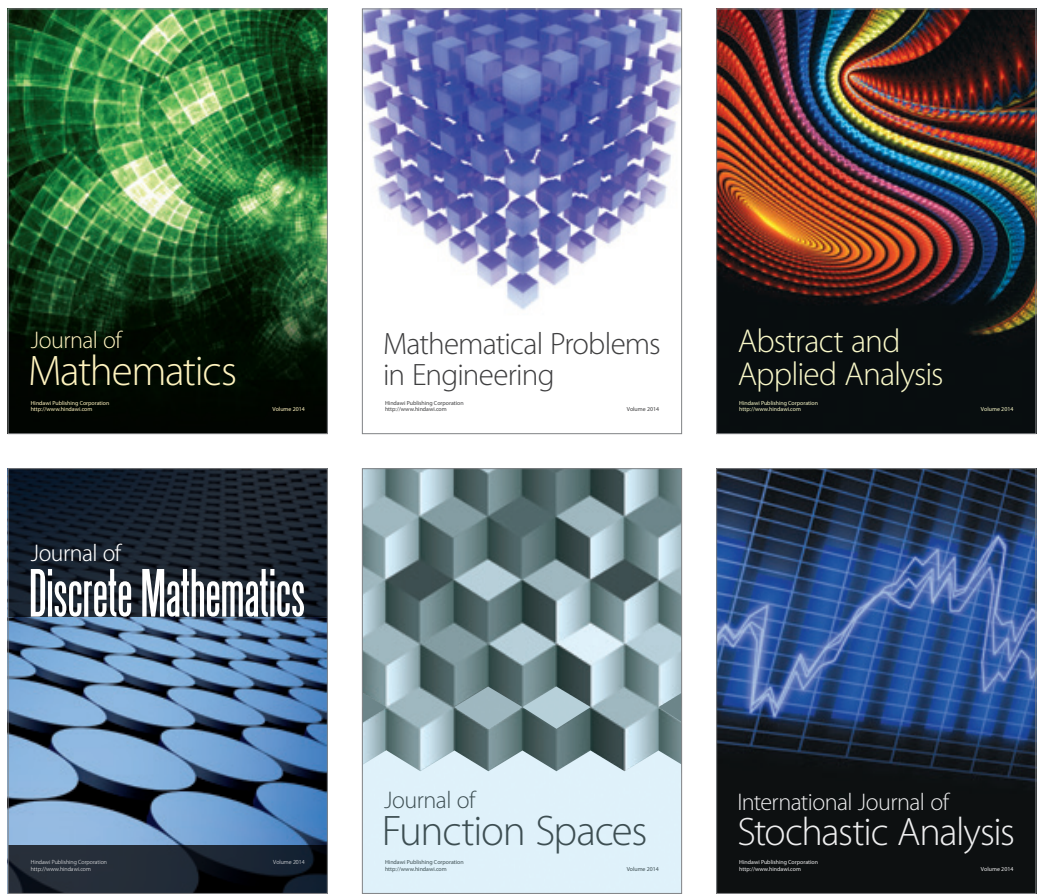

Journal of

Function Spaces

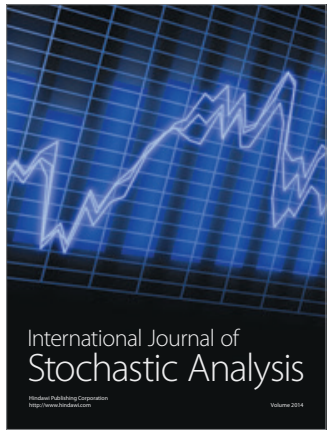

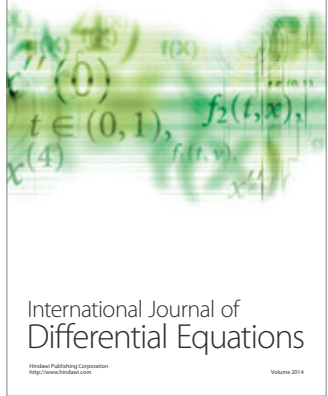
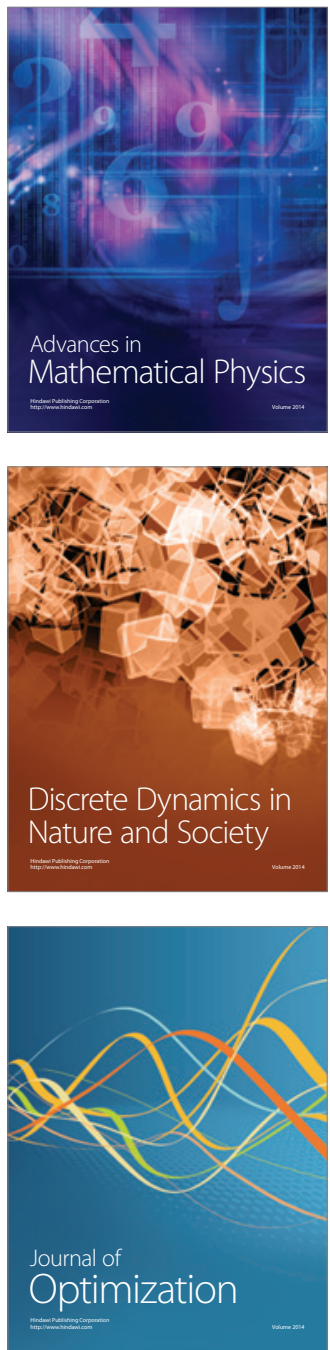